\title{
ERRATA
}

\section{Thermal and hydrostatic structure of the protoplanetary nebula exposed to stellar radiation and stellar wind from the central star}

Young Seok Yun, Hiroyuki Emori, and Kiyoshi Nakazawa

Earth Planets Space, Vol. 59, pp. 631-643, 2007

In the above-mentioned paper, we used an incorrect balance equation of force between the wind dynamical pressure and the nebular pressure on the contact surface by overlooking the change in the cross section for the momentum flux of the wind. Therefore, it is necessary to replace equations as follows.

1) Section 2. Equation (10):

$$
\text { "P=f } P_{\mathrm{w}} \quad \text { at } \quad z=z_{s}, " \text { should be replaced by } \quad " P=f^{2} P_{\mathrm{w}} \quad \text { at } \quad z=z_{s}, " .
$$

2) Section 2. Equation (15):

$$
\text { “ } f=M \eta^{\gamma+\frac{1}{4}} \sqrt{u} \exp \left(-N \frac{v^{2}}{\sqrt{\eta} u}\right), " \quad \text { should be replaced by } \quad “ f^{2}=M \eta^{\gamma+\frac{1}{4}} \sqrt{u} \exp \left(-N \frac{v^{2}}{\sqrt{\eta} u}\right), " .
$$

3) Section 2. Equation (18):

$$
\text { “ } \mathcal{L}(f, \eta)=1-\frac{f}{8 u^{4}}(1+2 s) " \quad \text { should be replaced by } \quad \text { “ } \mathcal{L}(f, \eta)=2-\frac{f}{8 u^{4}}(1+2 s) " .
$$

4) Appendix A. Equation (A.3):

$$
\text { “ } \mathcal{L}_{\eta}=-2 \zeta_{\mathrm{c}}^{2}-\left(2 \gamma-\frac{31}{4}\right) \zeta_{\mathrm{c}}-1, " \quad \text { should be replaced by } \quad “ \mathcal{L}_{\eta}=-2 \zeta_{\mathrm{c}}^{2}-\left(2 \gamma-\frac{63}{4}\right) \zeta_{\mathrm{c}}-2, ”
$$

5) Appendix A. Equation (A.4):

$$
\text { “ } \mathcal{L}_{f}=-2 \zeta_{\mathrm{c}}^{2}+10 \zeta_{\mathrm{c}}-1, " \text { should be replaced by } \quad \mathcal{L}_{f}=-2 \zeta_{\mathrm{c}}^{2}+20 \zeta_{\mathrm{c}}-2, " .
$$

6) Appendix A. Equation (A.5):

$$
" \mathcal{R}_{\eta}=\frac{1}{64 \zeta_{\mathrm{c}}\left(1-\zeta_{\mathrm{c}}\right)}\left\{-128 \zeta_{\mathrm{c}}^{4}-32(8 \gamma-15) \zeta_{\mathrm{c}}^{3}-2\left(32 \gamma^{2}-48 \gamma+257\right) \zeta_{\mathrm{c}}^{2}+16(\gamma+5) \zeta_{\mathrm{c}}+1\right\} "
$$

should be replaced by

$$
\text { " } \mathcal{R}_{\eta}=\frac{1}{16 \zeta_{\mathrm{c}}\left(2-\zeta_{\mathrm{c}}\right)}\left\{-32 \zeta_{\mathrm{c}}^{4}-8(8 \gamma-31) \zeta_{\mathrm{c}}^{3}-\left(16 \gamma^{2}-56 \gamma+\frac{961}{2}\right) \zeta_{\mathrm{c}}^{2}+8(\gamma+10) \zeta_{\mathrm{c}}+1\right\} " .
$$

7) Appendix A. Equation (A.6):

$$
\begin{aligned}
& \text { " } \mathcal{R}_{f}=2 \zeta_{\mathrm{c}}^{2}+\left(2 \gamma-\frac{31}{4}\right) \zeta_{\mathrm{c}}-\gamma-\frac{1}{8 \zeta_{\mathrm{c}}} . " \text { should be replaced by } \\
& " \mathcal{R}_{f}=2 \zeta_{\mathrm{c}}^{2}+\left(2 \gamma-\frac{63}{4}\right) \zeta_{\mathrm{c}}-\gamma-\frac{1}{4 \zeta_{\mathrm{c}}} . "
\end{aligned}
$$

Fortunately, the results on the temperature distribution and the height of the nebula hardly change even if the equations change. However, the behavior of solutions near the critical point changes. As a result, there exist surely the steady state solutions of the nebula, even if the wind parameter, $t_{*} / \beta$, is smallar than $1 \times 10^{7} \mathrm{yr}$ although the above-mentioned paper declared that there is no solution in such an extremely strong wind case. 
Figures 11 and 12 on p. 640 should read as follows:

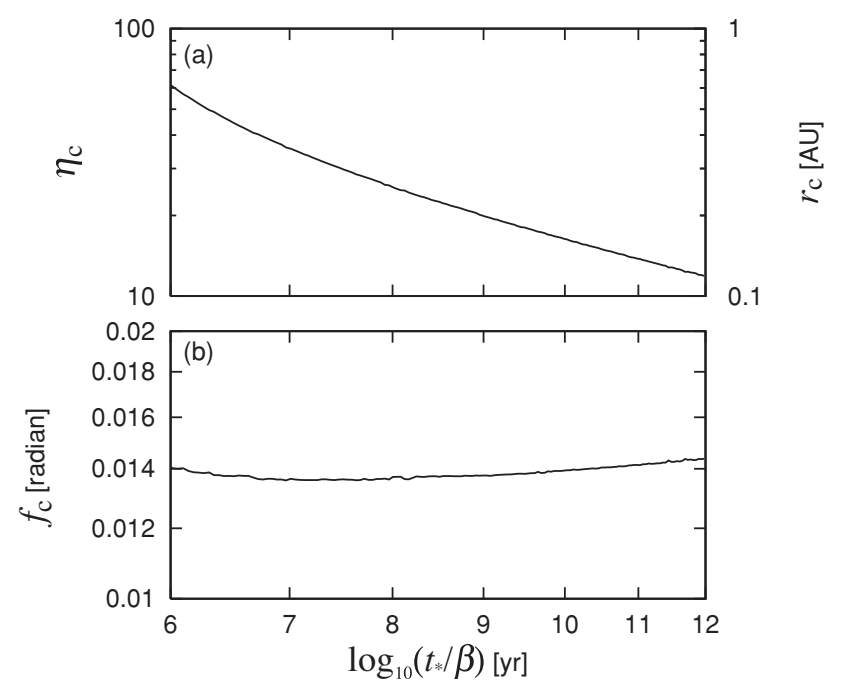

Fig. 11. Position of the critical point, $\eta_{\mathrm{c}}$ and $f_{\mathrm{c}}$, as a function of the wind parameter $t_{*} / \beta$.

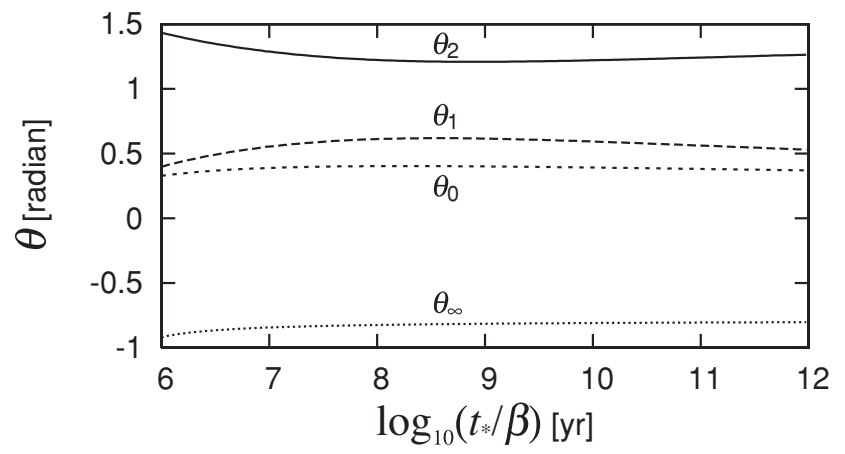

Fig. 12. Four characteristic angles $\theta_{0}, \theta_{\infty}, \theta_{1}$ and $\theta_{2}$ as functions of $t_{*} / \beta$. 